Journal of The Electrochemical Society, 151 (4) A571-A577 (2004)

\title{
Complex Capacitance Analysis of Porous Carbon Electrodes for Electric Double-Layer Capacitors
}

\author{
Jong H. Jang and Seung M. Oh*,Z \\ School of Chemical Engineering and Research Center for Energy Conversion and Storage, Seoul National \\ University, Seoul 151-744, Korea
}

\begin{abstract}
A new analytical methodology, complex capacitance analysis, is developed for porous carbons that are employed as electric double-layer capacitor (EDLC) electrodes. Based on the transmission line model, the imaginary capacitance profiles $\left(C_{\mathrm{im}} v s\right.$. $\left.\log f\right)$ are theoretically derived for a cylindrical pore and further extended to multiple pore systems. The theoretical derivation illustrates that two important electrochemical parameters in EDLCs can be estimated from the peak-shaped imaginary capacitance plots: total capacitance from the peak area and rate capability from the peak position. The usefulness of this analysis in estimating EDLC parameters is demonstrated by applying to two sets of practical porous carbon electrodes. In addition, the penetrability distribution curves that are derived from the experimental imaginary capacitance data using the log-normal assumption and discrete Fourier transform allow us to estimate the pore structure of carbon electrodes.
\end{abstract}

(C) 2004 The Electrochemical Society. [DOI: 10.1149/1.1647572] All rights reserved.

Manuscript submitted July 14, 2003; revised manuscript received October 28, 2003. Available electronically February 20,2004

The electric double-layer capacitor (EDLC) utilizes the double layer formed at the electrode/electrolyte interface where electric charges are accumulated on the electrode surface and ions of countercharge are arranged in the electrolyte side. The charge storable capacity of EDLCs that is normally expressed by capacitance is roughly proportional to electrode surface area, such that highsurface-area carbons are commonly utilized for EDLC electrodes. The rate capability of EDLCs is another important consideration for their practical application, because a complete utilization of electrode surface for charge storage is not always guaranteed. Incomplete utilization arises particularly at high-current conditions, under which ionic motions in the electrolyte are slower than the current transient. This undesirable situation is more pronounced when pores are narrow, long, and irregularly connected, as ions cannot penetrate into the deeper region, thereby the surface exposed there may not be utilized for charge storage. In short, the pore structure of EDLC electrodes is an important consideration because the capacitance is determined by surface area and the rate capability affected by pore size, length, and connectivity. ${ }^{1-6}$

Electrochemical impedance spectroscopy (EIS) has proven to be a useful tool for analysis of EDLC performance and pore structure of porous carbon electrodes. ${ }^{7-10}$ In previous studies, however, analyses were made mostly employing Nyquist plots $\left(-Z_{\text {im }} v s . Z_{\text {re }}\right)$ that are too featureless to obtain detailed information on pore structure. Sophisticated nonlinear fitting is also required to obtain EDLC data. Recently, there were several efforts to characterize the EDLC parameters using complex capacitance plots or frequency-dependent capacitance, but the detailed theoretical derivation was not given. ${ }^{6,11,12}$

In this paper we present a new analytical method that seems useful to estimate the EDLC performance of porous electrodes such as total capacitance and rate capability that are deeply related with the pore structure of porous electrodes. In the first half of this paper, the complex capacitance for a cylindrical pore and multipore systems is theoretically derived. Also, two methods for estimation of penetrability distribution patterns from experimental data are described. In the second half of this paper, application of the complex capacitance analysis made on two sets of porous carbon electrodes is provided.

\section{Theory}

Complex capacitance analysis for a cylindrical pore.-Porous electrodes have been analyzed with the transmission line model, which assumes the porous electrode surface as a distributed solid/

\footnotetext{
* Electrochemical Society Active Member.

z E-mail: seungoh@plaza.snu.ac.kr
}

liquid interface impedance with a variable ionic and electronic resistance according to the position of the interface from the pore opening. ${ }^{7-10,13-24}$ The overall impedance is derived as Eq. 1 with an assumption that the electrode resistance is negligible as compared to the ionic resistance in the electrolyte ${ }^{13}$

$$
Z=\left(R_{\text {ion }, \mathrm{L}} Z_{\mathrm{i}, \mathrm{L}}\right)^{1 / 2} \operatorname{coth}\left[\left(R_{\mathrm{ion}, \mathrm{L}} / Z_{\mathrm{i}, \mathrm{L}}\right)^{1 / 2} L\right]
$$

where $Z_{\mathrm{i}, \mathrm{L}}$ is the interfacial impedance per unit pore length, $R_{\text {ion, } \mathrm{L}}$ is the electrolyte resistance per unit pore length, and $L$ is the pore length. The elimination of electrode resistance is valid in an EDLC system, where common carbon materials have a higher electric conductivity ( $>10^{2} \mathrm{~S} \mathrm{~cm}^{-1}$ ) than electrolyte solutions (for example, $2.0 \mathrm{M}$ sulfuric acid: $\left.<1 \mathrm{~S} \mathrm{~cm}^{-1}\right){ }^{25,26}$ The interfacial impedance $\left(Z_{\mathrm{i}, \mathrm{L}}\right)$ can further be simplified as a purely capacitive one after ignoring the faradaic charge-transfer process that is pertinent to ideally polarized electrodes, for example, carbon electrodes for EDLC application. The $Z_{\mathrm{i}, \mathrm{L}}$ and $R_{\mathrm{ion}, \mathrm{L}}$ for a cylindrical pore are then given as

$$
Z_{\mathrm{i}, \mathrm{L}}(f)=\frac{L}{j 2 \pi f C_{0}}=\frac{1}{2 \pi r} \frac{1}{j 2 \pi f C_{\mathrm{d}}}, \quad R_{\text {ion, } \mathrm{L}}=\frac{1}{\kappa \pi r^{2}}
$$

The impedance of porous electrodes is then expressed as

$$
\begin{gathered}
Z(\alpha)=R_{0} \times \alpha \sqrt{2 / j} \operatorname{coth}\left[(\alpha \sqrt{2 / j})^{-1}\right] \\
\alpha=\alpha_{0} \omega^{-0.5}=\frac{\alpha_{0}}{\sqrt{2 \pi f}}, \quad \text { where } \alpha_{0}=\frac{1}{2} \sqrt{\frac{\kappa}{C_{\mathrm{d}}}} \frac{\sqrt{r}}{L}=\frac{1}{\sqrt{2 R_{0} C_{0}}}
\end{gathered}
$$

Here, $C_{0}\left(=C_{\mathrm{d}} \times 2 \pi r L\right)$ and $R_{0}\left(=R_{\text {ion, } \mathrm{L}} \times L\right)$ are the capacitance and electrolyte resistance for the entire pore, respectively. The dimensionless penetration depth $(\alpha)$ is defined in this work as a function of frequency $(f)$, and the penetrability $\left(\alpha_{0}\right)$ that is in turn defined by the pore structure ( $r$ : pore radius, $L$ : pore length) of electrodes and electrochemical parameters (к: electrolyte conductivity, $C_{\mathrm{d}}$ : double-layer capacitance per unit area) in pores. The dimensionless penetration depth reflects the degree of ion penetration into pores according to the current transient; thereby it is an important parameter to characterize the rate capability of EDLC electrodes. When the current transient is rapidly varied at high ac frequencies, $\alpha$ becomes smaller because ions cannot penetrate deep inside pores due to the limited ionic motions. A smaller $\alpha$ is also expected if either the pore radius is smaller or pore length is longer because the degree of ion penetration becomes lower.

The complex capacitance for a cylindrical pore is calculated from Eq. 3 as 

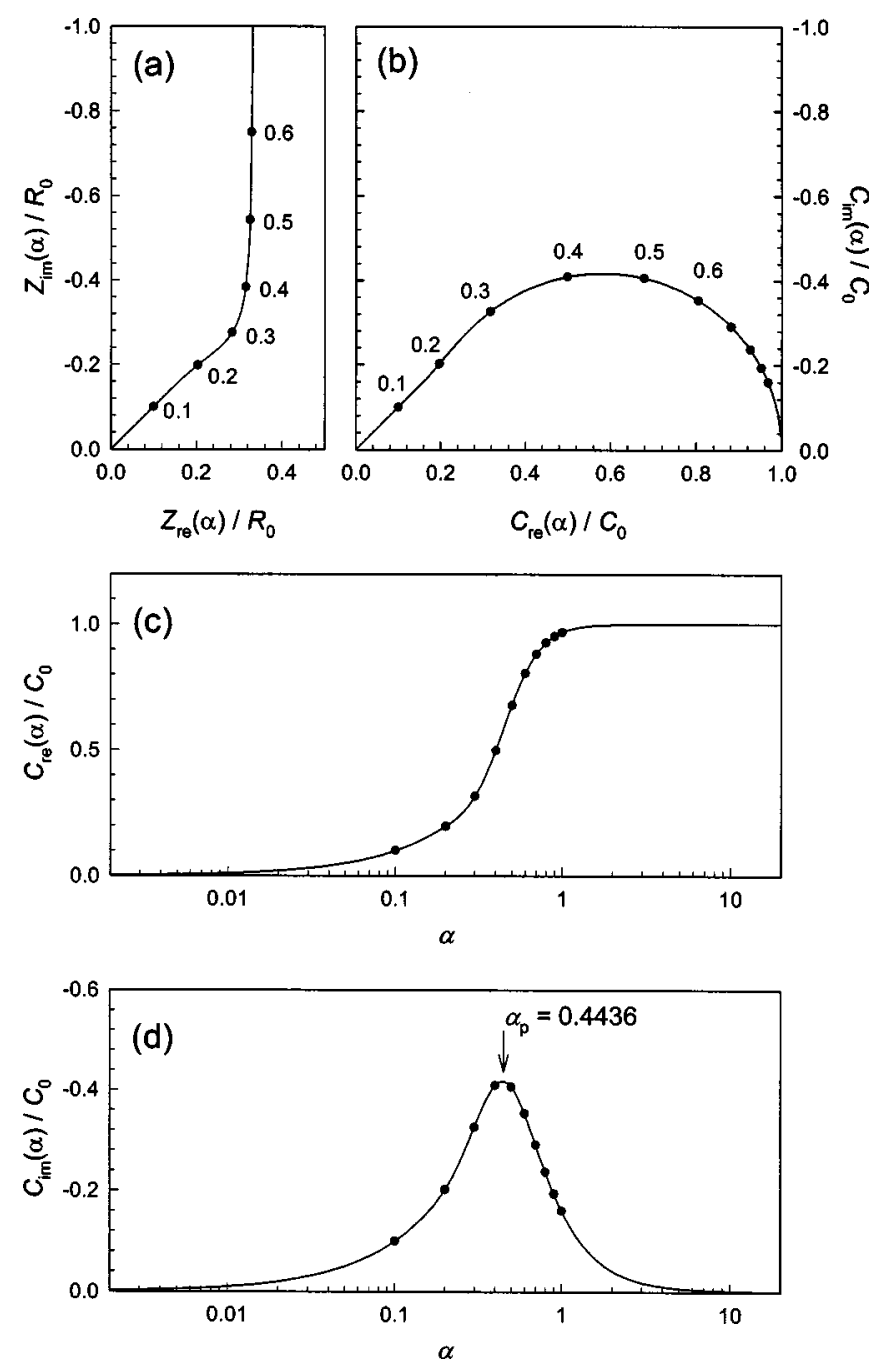

Figure 1. Simulated Nyquist plots for a cylindrical pore: (a) impedance, (b) complex capacitance, (c) the real part, and (d) the imaginary part of complex capacitance as a function of dimensionless penetrability $(\alpha)$. The numbers in the figures are $\alpha$ values.

$$
C(\alpha)=\frac{1}{j \omega Z(\alpha)}=C_{0} \times \alpha \sqrt{2 / j} \tanh \left[(\alpha \sqrt{2 / j})^{-1}\right]
$$

Then, the real and imaginary part of the complex capacitance is derived as follows

$$
\begin{gathered}
C_{\mathrm{re}}(\alpha)=C_{0} \times\left(\alpha \frac{\sinh \alpha^{-1}+\sin \alpha^{-1}}{\cosh \alpha^{-1}+\cos \alpha^{-1}}\right) \\
C_{\mathrm{im}}(\alpha)=C_{0} \times\left(-\alpha \frac{\sinh \alpha^{-1}-\sin \alpha^{-1}}{\cosh \alpha^{-1}+\cos \alpha^{-1}}\right)=C_{0} C_{\mathrm{im}}^{0}(\alpha)
\end{gathered}
$$

In Fig. 1a, the Nyquist impedance plot that is simulated on a cylindrical pore using Eq. 3 is presented. The curve shape is changed with an increase in $\alpha$ from a straight line with $45^{\circ}$ slope, intermediate region, and vertical line. As seen, the profile is so featureless that a visual estimation of the EDLC properties seems difficult. The complex capacitance plot that is derived using Eq. 5 is displayed in Fig. 1b, and the real $\left(C_{\mathrm{re}}\right)$ and imaginary part $\left(C_{\mathrm{im}}\right)$ of complex capacitance that are calculated using Eq. 6 and 7, respectively, are plotted as a function of $\alpha$ in Fig. $1 \mathrm{c}$ and d. $C_{\mathrm{re}}$ increases
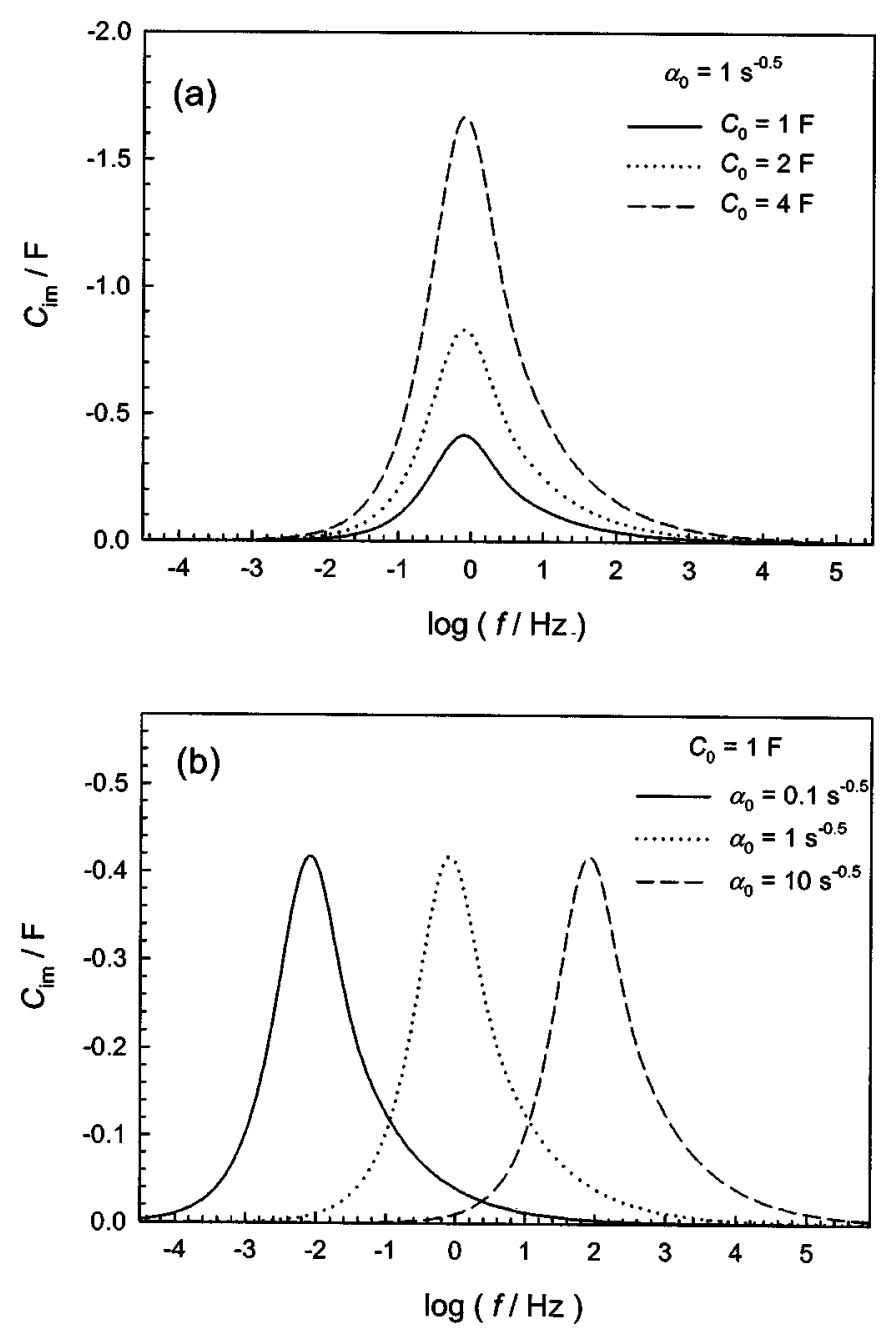

Figure 2. (a) Relationship between the total capacitance $\left(C_{0}\right)$ of a cylindrical pore and peak area in the imaginary capacitance plot (penetrability is assumed the same at $\left.1 \mathrm{~s}^{-0.5}\right)$. (b) Relationship between the penetrability $\left(\alpha_{0}\right)$ and peak position in an imaginary capacitance plot (total capacitance is assumed the same at $1 \mathrm{~F}$ ).

monotonically from 0 to $C_{0}$ with $\alpha$ (Fig. 1c), whereas $C_{\text {im }}$ shows a peak-shaped curve with a maximum at $\alpha_{\mathrm{p}}=0.4436$ (Fig. 1d). The total capacitance can be estimated from the $C_{\mathrm{re}}$ value at a sufficiently high $\alpha$ (low-frequency extreme). In practice, however, it is difficult to obtain data at such a low frequency due to the instrumental limitation and extremely long measuring time. In contrast, when the imaginary capacitance plot is utilized, total capacitance can be estimated by simply integrating the peak area. The quantitative relationship between the peak area $(A)$ and total capacitance $\left(C_{0}\right)$ is obtained as

$$
A=-\int_{-\infty}^{\infty} C_{\mathrm{im}}(\alpha) d \log f=0.682 C_{0}
$$

The factor, 0.682 , obtained by a numerical integration of $C_{\text {im }}(\alpha)$ in Eq. 7 is identical to $\pi /(2 \ln 10)$ that is predicted from KramersKronig transforms. ${ }^{27,28}$ The imaginary capacitance plots calculated with a variation in $C_{0}$ are presented in Fig. 2a. As seen, the peak area is proportional to the total capacitance as expected from Eq. 8.

The peak frequency $\left(f_{\mathrm{p}}\right)$ where the imaginary capacitance has a maximum value can be obtained by substituting $\alpha$ with $\alpha_{\mathrm{p}}$ in Eq. 4 as 


$$
f_{\mathrm{p}}=\frac{1}{2 \pi \alpha_{\mathrm{p}}^{2}} \alpha_{0}^{2}=0.809 \alpha_{0}^{2}
$$

The change of $f_{\mathrm{p}}$ according to $\alpha_{0}$ is demonstrated in Fig. 2b, where it is seen that the two parameters are correlated to each other as described in Eq. 9. The peak of the same area, as $C_{0}$ is assumed the same, moves to the high-frequency direction when $\alpha_{0}$ becomes higher.

Complex capacitance analysis for multiple pore systems.-As the porous carbons used in practical EDLCs contain many nonuniform pores that differ in both radius and length, the penetrability should have a certain distribution instead of a single value. In multiple-pore systems, the capacitance $\left(C_{0, \mathrm{i}}\right)$ and penetrability $\left(\alpha_{0, \mathrm{i}}\right)$ of the $i$ th pore are

$$
C_{0, \mathrm{i}}=C_{\mathrm{d}, \mathrm{i}}\left(2 \pi r_{\mathrm{i}} L_{\mathrm{i}}\right), \quad \alpha_{0, \mathrm{i}}=\frac{1}{2} \sqrt{\frac{\kappa_{\mathrm{i}}}{C_{\mathrm{d}, \mathrm{i}}}} \frac{\sqrt{r_{\mathrm{i}}}}{L_{\mathrm{i}}}
$$

where $\kappa_{\mathrm{i}}, C_{\mathrm{d}, \mathrm{i}}, r_{\mathrm{i}}$, and $L_{\mathrm{i}}$ are the electrolyte conductivity, doublelayer capacitance per unit area, pore radius, and pore length of the $i$ th pore, respectively. Then the frequency-dependent imaginary capacitance is calculated as

$$
\begin{aligned}
C_{\mathrm{im}}(f) & =\sum_{\mathrm{i}} C_{\mathrm{im}}^{0}\left(\alpha_{0, \mathrm{i}}, f\right) C_{0, \mathrm{i}} \\
& =\int_{0}^{\infty} C_{\mathrm{im}}^{0}\left(\frac{\alpha_{0}}{\sqrt{2 \pi f}}\right) C_{\mathrm{tot}}\left[p\left(\alpha_{0}\right) d \alpha_{0}\right] \\
& =\int_{-\infty}^{\infty} C_{\mathrm{im}}^{0}\left(\frac{\alpha_{0}}{\sqrt{2 \pi f}}\right) C_{\mathrm{tot}}\left[p^{\prime}\left(\alpha_{0}\right) d \log \alpha_{0}\right] \\
p\left(\alpha_{0}\right)= & \frac{d\left[C\left(\alpha_{0}\right) / C_{\mathrm{tot}}\right]}{d \alpha_{0}}, \quad p^{\prime}\left(\alpha_{0}\right)=\frac{d\left[C\left(\alpha_{0}\right) / C_{\mathrm{tot}}\right]}{d \log \alpha_{0}}
\end{aligned}
$$

where $C\left(\alpha_{0}\right)$ represents the sum of $C_{0, \mathrm{i}}$ for pores having the same penetrability of $\alpha_{0}$, whereas $C_{\text {tot }}$ is the sum of $C\left(\alpha_{0}\right)$ for entire pores, and $p\left(\alpha_{0}\right)$ and $p^{\prime}\left(\alpha_{0}\right)$ are the $\alpha_{0}$ distribution function in linear and logarithmic scale, respectively. The total capacitance $\left(C_{\text {tot }}\right)$ is related to the peak area by integrating Eq. 11 with $\log f$ as

$$
\begin{aligned}
A & =-\int_{-\infty}^{\infty} C_{\mathrm{im}}(f) d \log f \\
& =-\left(\int_{-\infty}^{\infty} C_{\mathrm{im}}^{0}(\alpha) d \log f\right)\left(\int_{0}^{\infty} C_{\mathrm{tot}} p\left(\alpha_{0}\right) d \alpha_{0}\right) \\
& =0.682 C_{\mathrm{tot}}
\end{aligned}
$$

In this work, the characteristic penetrability $\left(\alpha_{0}^{*}\right)$ has been defined as the $\alpha_{0}$ value having a maximum population in penetrability distribution curves. This parameter is expressed as a function of peak frequency and an empirical parameter $\beta$ as

$$
\alpha_{0}^{*}=\sqrt{2 \pi} \alpha_{\mathrm{p}} \beta f_{\mathrm{p}}^{0.5}=1.11 \beta f_{\mathrm{p}}^{0.5}
$$

The $\beta$ is inserted because the peak frequency is different from that calculated by Eq. 9 with $\alpha_{0}^{*}$ due to the unsymmetric nature of the $C_{\mathrm{im}}^{0}(\alpha)$ function. $\beta$ varies according to the broadness of the penetrability distribution curves. As the distribution becomes narrower, $\beta$ approaches unity and Eq. 14 is simplified to Eq. 9, which is relevant to a single-pore system.

Total capacitance $\left(C_{\text {tot }}\right)$ and penetrability $\left(\alpha_{0}\right)$ distribution in multiple-pore systems.-As described in Eq. 4 , the penetrability in multipore systems has a certain distribution because the four param-
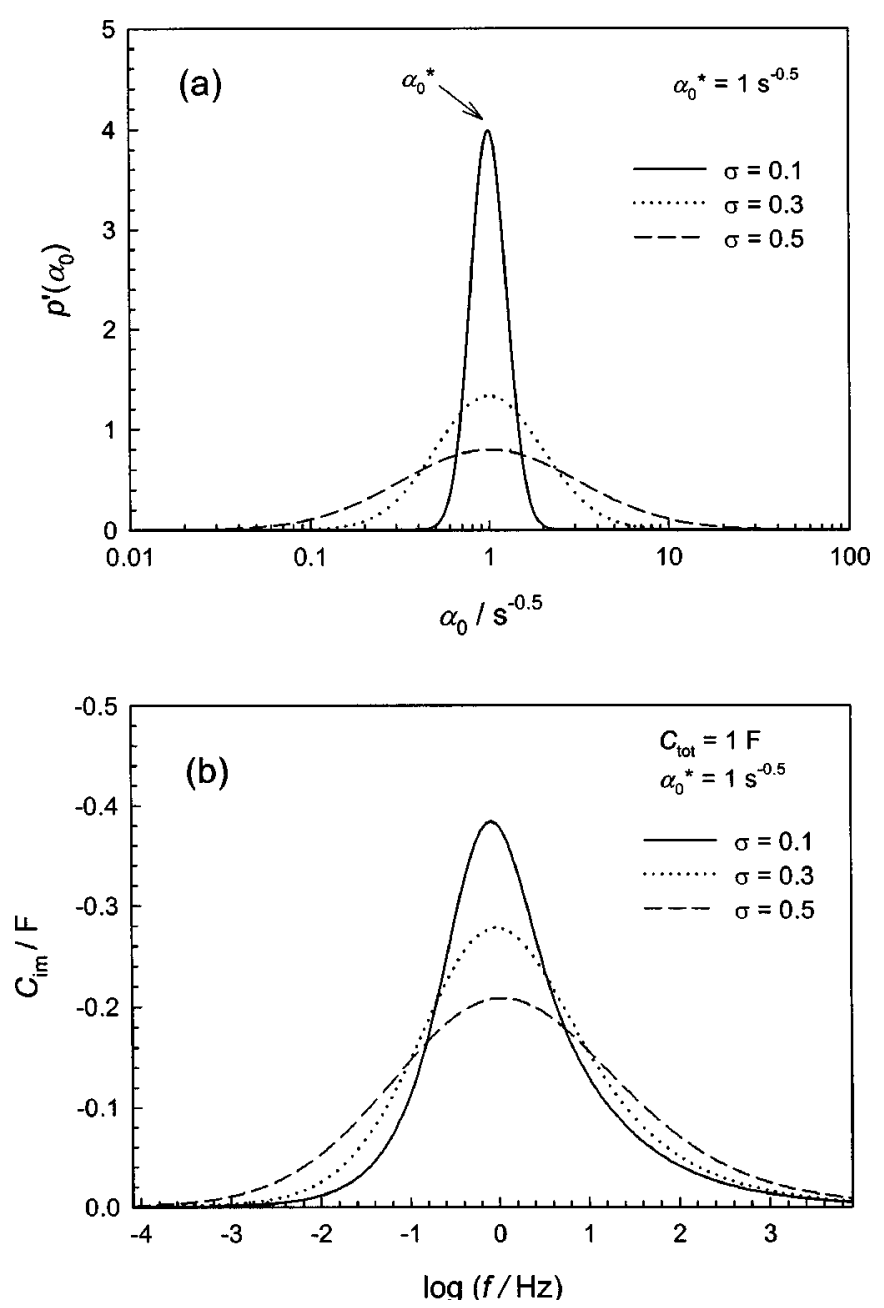

Figure 3. (a) Log-normal $\alpha_{0}$ distribution as a function of standard deviation $(\sigma)$ and $(b)$ the resultant imaginary capacitance plots.

eters $\left(r, L, \kappa\right.$, and $\left.C_{\mathrm{d}}\right)$ are variable according to pore structure. The total capacitance and $\alpha_{0}$ distribution pattern in multipore systems can be obtained from experimental capacitance data by solving Eq. 11 , where $C_{\mathrm{im}}(f)$ is the experimentally observed imaginary capacitance data. To solve Eq. 11, two approaches are made in this work.

In the first approach, $\alpha_{0}$ is assumed to have a log-normal distribution; thereby the ultimate goal here is the extraction of the degree of dispersion as the distribution pattern is preassumed. This assumption is made as porous materials frequently show a log-normal distribution in pore size. ${ }^{8,9}$ The log-normal $\alpha_{0}$ distribution function is expressed by two parameters: standard deviation $(\sigma)$ that reflects the degree of dispersion and characteristic penetrability $\left(\alpha_{0}^{*}\right)$ as

$$
\begin{aligned}
p^{\prime}\left(\alpha_{0}\right) & =\frac{d\left[C\left(\alpha_{0}\right) / C_{\text {tot }}\right]}{d \log \alpha_{0}} \\
& =\frac{1}{\sqrt{2 \pi} \sigma} \exp \left[-\frac{1}{2 \sigma^{2}}\left(\log \alpha_{0}-\log \alpha_{0}^{*}\right)^{2}\right]
\end{aligned}
$$

To obtain the standard deviation from experimental data, the polynomial interpolation method was utilized. As the first step in this method, log-normal $\alpha_{0}$ distributions are derived using Eq. 15 as a function of $\sigma$ with $\alpha_{0}^{*}\left(1 \mathrm{~s}^{-0.5}\right)$ being fixed (Fig. 3a). Then the imaginary capacitance is calculated using Eq. 11. In Fig. 3b, three imaginary capacitance plots that are obtained with three different $\sigma$ are represented. In the profiles, the peak width increases at the ex- 

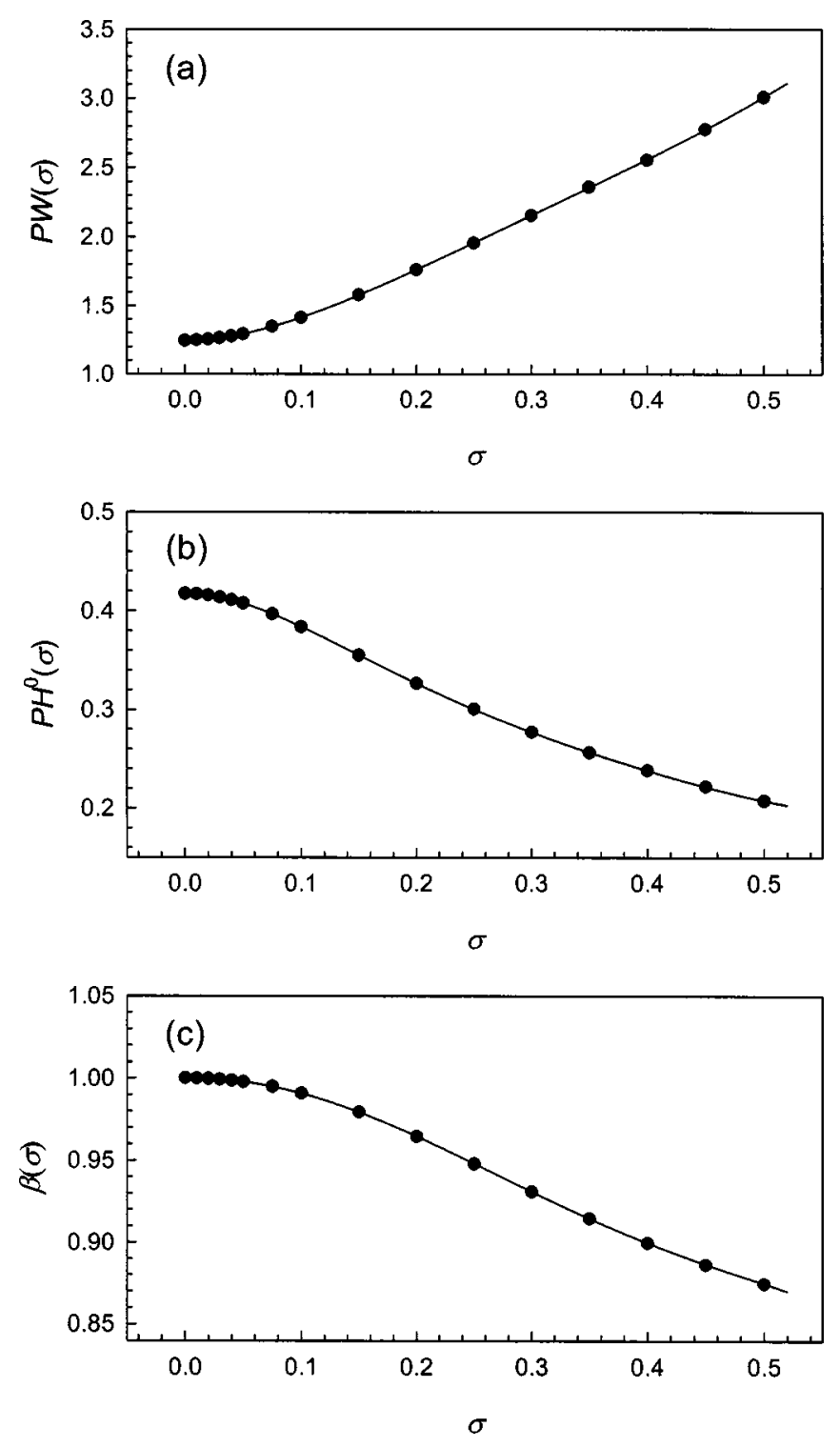

Figure 4. (a) The peak width $(P W)$, (b) peak height $\left(P H^{0}\right)$, and (c) $\beta$ in imaginary capacitance plots as a function of standard deviation $(\sigma)$ in lognormal $\alpha_{0}$ distribution. The data points are derived from 16 imaginary capacitance plots and the lines indicate the best fitting with 5th-order polynomial functions.

pense of peak height when $\sigma$ increases. Also note that the peak area remains unchanged as $C_{\text {tot }}$ is fixed at a constant value. The peak frequency slightly moves to a higher value due to a decrease in $\beta$ as $\sigma$ increases. The next step is the location of the peak width (the full width at half maximum, fwhm, $P W$ ), peak height $\left(P H^{0}\right)$, and $\beta$ from the calculated imaginary capacitance plots. The $P W, P H^{0}$, and $\beta$ that are collected from sixteen imaginary capacitance plots that are made with sixteen different $\sigma$ are indicated as the closed circles in Fig. 4. The data points are best-fitted with 5th-order polynomial functions

$$
\begin{aligned}
P W(\sigma)= & 1.2453-2.21 \times 10^{-3} \sigma+22.0 \sigma^{2}-58.5 \sigma^{3} \\
& +72.0 \sigma^{4}-29.2 \sigma^{5} \\
P H^{0}(\sigma)= & 0.4173-0.012 \sigma-5.11 \sigma^{2}+19.5 \sigma^{3}-31.8 \sigma^{4} \\
& +19.7 \sigma^{5}
\end{aligned}
$$

$$
\begin{aligned}
\beta(\sigma)= & 1.00+3.48 \times 10^{-3} \sigma-0.936 \sigma^{2}-0.889 \sigma^{3} \\
& +6.54 \sigma^{4}-6.10 \sigma^{5}
\end{aligned}
$$

Now the standard deviation can be obtained from given experimental impedance data. To this end, the peak frequency $\left(f_{\mathrm{p}}\right)$, peak height $(P H)$, and peak width $(P W)$ should roughly be decided from the experimental imaginary capacitance plot. Then $\sigma$ is obtained by interpolating the $P W$ value in Fig. 4 a. $C_{\text {tot }}$ is calculated by dividing the peak height $(P H)$ by $P H^{0}(\sigma)$, which corresponds to the peak height when $C_{\text {tot }}=1 \mathrm{~F} . \alpha_{0}^{*}$ is calculated from the peak frequency and $\beta(\sigma)$ using Eq. 14. The $\alpha_{0}$ distribution is then derived using Eq. 15 with as-obtained $\sigma$ and $\alpha_{0}^{*}$ values. The $C_{\text {tot }}$ and $\alpha_{0}$ distribution that are obtained so far still need refinement because they are based on the roughly estimated $f_{\mathrm{p}}, P H$, and $P W$ values from the experimental data points. The refinement is made by fitting the experimental data with the simulated imaginary capacitance profiles obtained from the as-obtained $C_{\text {tot }}$ and $\alpha_{0}$ distribution with Eq. 11. The three parameters $\left(C_{\text {tot }}, \sigma, \alpha_{0}^{*}\right)$ are extracted from the best fitting, from which the final $C_{\text {tot }} p^{\prime}\left(\alpha_{0}\right)$ curves are derived using Eq. 15.

The second approach to obtain $C_{\text {tot }}$ and the $\alpha_{0}$ distribution is the use of the discrete Fourier transform (DFT). This method seems more versatile than the previous one because it can be applied to any type of $\alpha_{0}$ distribution, but a noise reduction procedure as well as complicated transformations is required to obtain a reasonable simulation. Equation 11 can be converted to a convolution form using two defined functions: $x=\ln (2 \pi f)$ and $y=\ln \alpha_{0}^{2}$

$$
g(x)=\int_{-\infty}^{\infty} h(x-y) f(y) d y=(h * f)(x)
$$

with

$$
\begin{gathered}
f(y)=C_{\mathrm{tot}} \frac{d\left[C\left(\alpha_{0}\right) / C_{\mathrm{tot}}\right]}{d \ln \alpha_{0}^{2}}, \quad g(x)=C_{\mathrm{im}}(x), \\
h(x-y)=C_{\mathrm{im}}^{0}\left(\alpha=e^{-(x-y) / 2}\right)
\end{gathered}
$$

where $f(x), g(x)$, and $h(x)$ represent the $\alpha_{0}$ distribution function, experimental imaginary capacitance, and known transfer function, respectively. The DFT and inverse transform are defined as ${ }^{29,30}$

$$
F_{\mathrm{k}}=\frac{1}{N} \sum_{-\mathrm{N} / 2+1}^{\mathrm{N} / 2} f_{\mathrm{n}} \mathrm{e}^{-\mathrm{i} 2 \pi \mathrm{nk} / \mathrm{N}}, \quad f_{n}=\sum_{-\mathrm{N} / 2+1}^{\mathrm{N} / 2} F_{\mathrm{k}} \mathrm{e}^{\mathrm{i} 2 \pi \mathrm{nk} / \mathrm{N}}
$$

where $f_{n}$ and $F_{\mathrm{k}}$ are the original data set and discrete Fourier transformed one, respectively, and $N$ is the number of data points. Equation 17 is discrete Fourier transformed to

$$
G_{\mathrm{k}}(v)=N \Delta x H_{\mathrm{k}}(v) F_{\mathrm{k}}(v)
$$

where $\Delta x$ is the data interval and $v$ is the reciprocal $x$. Then, the $\alpha_{0}$ distribution is calculated according to the following order: $(i)$ discrete Fourier transforming experimentally determined $g(x)$ and known $h(x)$, (ii) dividing $G_{\mathrm{k}}(v)$ by $H_{\mathrm{k}}(v)$ to yield $F_{\mathrm{k}}(v)$, and (iii) inverse transforming to determine $f(x)$. The procedure to obtain the $\alpha_{0}$ distribution from a given experimental imaginary capacitance is as follows. The three profiles shown in Fig. 3b are taken as the experimental data. The experimental imaginary capacitance profiles are discrete Fourier transformed to $G_{\mathrm{k}}(v)$ (Fig. 5a), which are divided by $N \Delta x H_{\mathrm{k}}(v)$ to yield $F_{\mathrm{k}}(v)$ as shown in Fig. 5 c. The inverse transformation of $F_{\mathrm{k}}(v)$ produces $\alpha_{0}$ distributions, which are identical to the initial log-normal ones (Fig. 3a).

\section{Experimental}

The EDLC performance of porous carbon electrodes was examined using the complex capacitance analysis. The molecular-sieving 

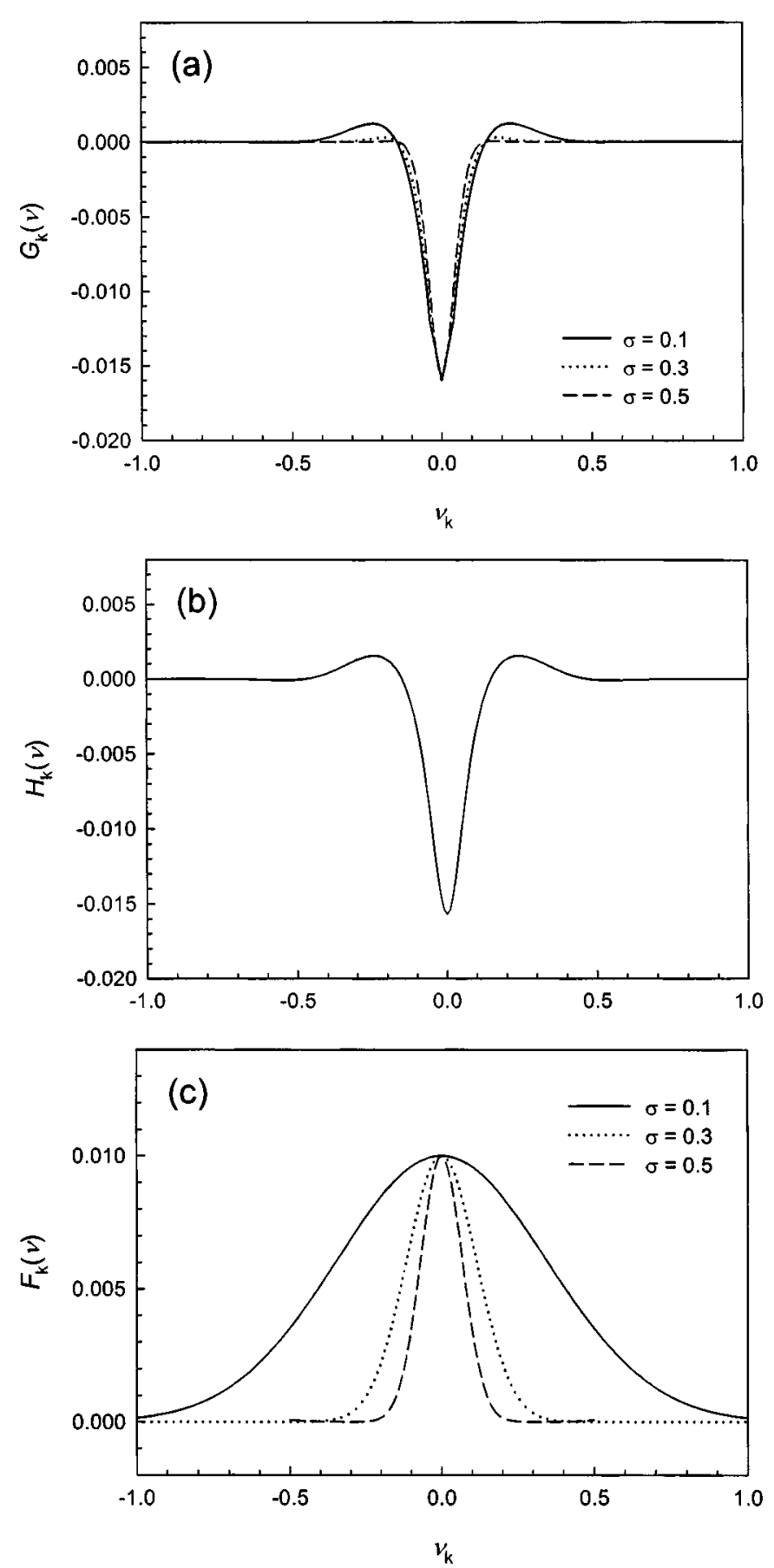

Figure 5. DFT profiles for (a) experimental imaginary capacitance, (b) transfer function, and (c) $\alpha_{0}$ distribution calculated from (a) and (b). The inverse transformation of (c) generates an $\alpha_{0}$ distribution that is identical to Fig. 3a, thus omitted.

carbon (MSC25) was provided by Kansai Coke and Chemicals Co. and new mesoporous carbon (NMC) was prepared using the Mobile Composite Material 48 as a template. ${ }^{4,31}$ The other series of mesoporous carbons were prepared by using a silica-sol template. ${ }^{28}$ The average pore diameter of these carbons is similar $(c a .9 \mathrm{~nm})$ because identical silica sol particles were used to generate mesopores, but the pore volume differs as the silica-sol content (the mole ratio of silica to carbon precursor: $\mathrm{S} 1=0.77 ; \mathrm{S} 2=1.16 ; \mathrm{S} 3=1.54$ ) was adjusted to different values. The surface area and pore size distribution were calculated from the $\mathrm{N}_{2}$ adsorption isotherms (Micromet-
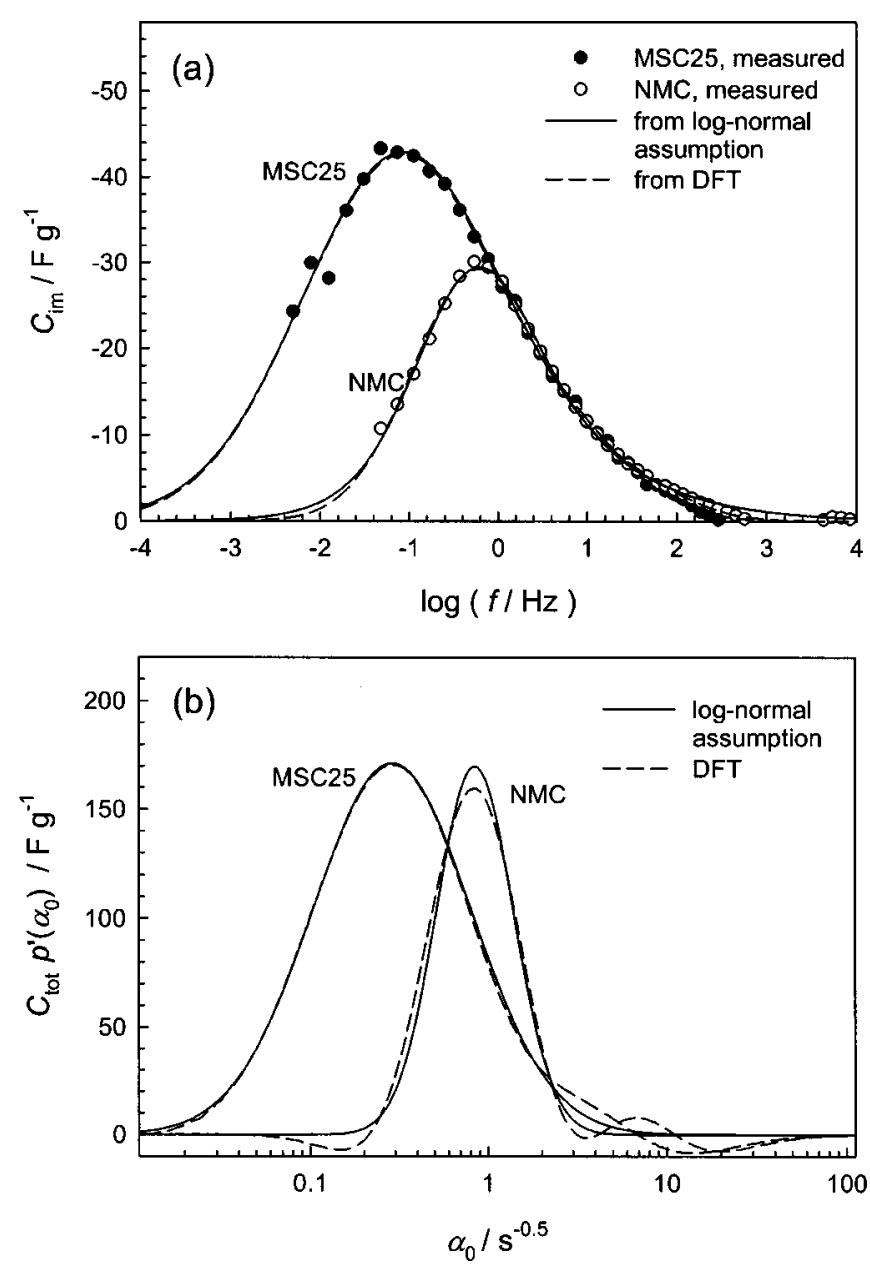

Figure 6. (a) Imaginary capacitance plots converted from experimental impedance data for MSC25 and NMC electrodes. The lines indicate the imaginary capacitance curves that are best-fitted to the experimental data points. (b) $C_{\text {tot }} p^{\prime}\left(\alpha_{0}\right)$ curves for MSC25 and NMC by log-normal assumption (solid line) and DFT (dashed line).

rics ASAP 2010) using the Brunauer, Emmett, and Teller (BET), and Barrett, Joyner, and Halenda (BJH) method, respectively.

A three-electrode configuration was used with a platinum flag as the counter electrode and saturated calomel electrode (SCE) as the reference electrode. For the preparation of working electrodes, a mixture of carbon power, polytetrafluoroethylene (PTFE) binder, and carbon additive for conductivity enhancement (Ketjenblack ECP-600JD) was coated on a stainless steel mesh (apparent area 2 $\mathrm{cm}^{2}$ ). The weight of carbon powder was $10 \mathrm{mg}$. The loading amount of carbon additive was controlled to give a comparable electronic conductivity for each electrode. The electrolyte was $2.0 \mathrm{M}$ sulfuric acid. EIS measurements were made over the frequency range of $10^{6}-5 \times 10^{-3} \mathrm{~Hz}$ (Zahner, im6e) with ac amplitude of $10 \mathrm{mV}$. Experimental data were processed and analyzed with Mathcad 2000 Professional (MathSoft, Inc.) software.

\section{Results and Discussion}

In Fig. 6a, the imaginary capacitance data that are converted from the experimentally observed impedance data for MSC25 and NMC are indicated as the closed and open circles, respectively. Even with the raw data points, one can compare at first glance the EDLC performance of the two carbon electrodes. The area under the peak-shaped profile of MSC25 is larger than that of NMC, indicative of a larger total capacitance in the former. It is also predictable that the NMC electrode has a better rate capability than that of MSC25 as the peak frequency is higher. A more quantitative analysis is made 


\begin{tabular}{|c|c|c|}
\hline & MSC25 & NMC \\
\hline$C_{\text {tot }}\left(\mathrm{F} \mathrm{g}^{-1}\right)$ & $188.9 \pm 2.2$ & $92.8 \pm 0.6$ \\
\hline$\sigma$ & $0.440 \pm 0.011$ & $0.218 \pm 0.005$ \\
\hline$\alpha_{0}^{*}\left(s^{-0.5}\right)$ & $0.288 \pm 0.008$ & $0.831 \pm 0.003$ \\
\hline$A_{\mathrm{BET}}\left(\mathrm{m}^{2} \mathrm{~g}^{-1}\right)^{\mathrm{a}}$ & 1970 & 1257 \\
\hline$D_{\text {pore,aver. }}(\mathrm{nm})^{\mathrm{b}}$ & $<2.0$ & ca. 2.3 \\
\hline
\end{tabular}

after a derivation of $\alpha_{0}$ distribution curves for the two electrodes (Fig. 6b). Two features are apparent in Fig. 6b. First, the two methods give largely the same penetrability distribution for the MSC25 electrode and also for the NMC electrode, even if there appears some mismatch. This implies that the log-normal assumption in $\alpha_{0}$ distribution is valid for the two porous carbons. Secondly, MCS25 gives rise to smaller $\alpha_{0}$ values as compared to those for NMC, illustrating that the pores of either smaller radius or longer length are more populated in MSC25. The narrower $\alpha_{0}$ distribution in NMC suggests that the pores in this carbon are more uniform either in radius or length. The penetrability is a function of both pore structure and electrochemical parameters as expressed in Eq. 4, such that the prediction of pore structure from $\alpha_{0}$ distribution is only possible when the electrochemical parameters are known. The electrolyte conductivity $(\kappa)$ in pores differs to that of bulk; furthermore, it varies according to the pore size. The double-layer capacitance per unit area $\left(C_{\mathrm{d}}\right)$ is also varied according to the pore size and surface properties of pores. Even with this uncertainty, it may be safe to say that two electrochemical parameters $\left(\kappa\right.$ and $C_{\mathrm{d}}$ ) are similar in the two electrodes as their pore size is comparable to each other in the range of 1-2 nm. It should further be noted that the pore radius and length cannot be predicted separately but as a combination of the two.

The imaginary capacitance profiles that are best-fitted to the experimental data are indicated as lines in Fig. 6a. The profiles derived from the log-normal assumption and Fourier transform look largely the same and are reasonably fitted to the experimental data points. The total capacitance $\left(C_{\text {tot }}\right)$ and characteristic penetrability $\left(\alpha_{0}^{*}\right)$ are obtained from the best-fitted imaginary capacitance profiles using Eq. 11 and 15, and the results are listed in Table I. The former can also be calculated from the peak area $(A)$ and the latter from the peak frequency $\left(f_{\mathrm{p}}\right)$ using Eq. 13 and 14, respectively. The EDLC data derived from the complex capacitance analysis are reasonably matched to those predictable from gas adsorption analysis. The higher total capacitance in MSC25 is ascribed to its larger surface area. The smaller $\alpha_{0}$ in MSC25 is due to the dominance of micropores $(<2.0 \mathrm{~nm})$, which is compared to the NMC electrode that contains mesopores (average diameter $2.3 \mathrm{~nm}$ ).

The complex capacitance analysis is made for another series of carbon electrodes that were prepared using silica-sol template. In Fig. 7a, the experimental imaginary capacitance data for three carbon electrodes are indicated as circles or triangles. It is immediately apparent even before the fitting that the S3 electrode has the largest total capacitance and best rate capability among the three as predicted from its peak area and peak frequency. The simulated imaginary capacitance profiles that are best-fitted to the experimental data with an assumption of log-normal $\alpha_{0}$ distribution are indicated as lines in Fig. 7a, and the $C_{\text {tot }} p^{\prime}\left(\alpha_{0}\right)$ curves that are derived from the best-fitted imaginary capacitance profiles using Eq. 11 are presented in Fig. 7b. The total capacitance $\left(C_{\text {tot }}\right)$ and characteristic penetrability $\left(\alpha_{0}^{*}\right)$ that are obtained with the best-fitting are listed in Table II. S3 shows the largest total capacitance among the three, from which it is predicted that $\mathrm{S} 3$ has the largest surface area. The gas adsorp-
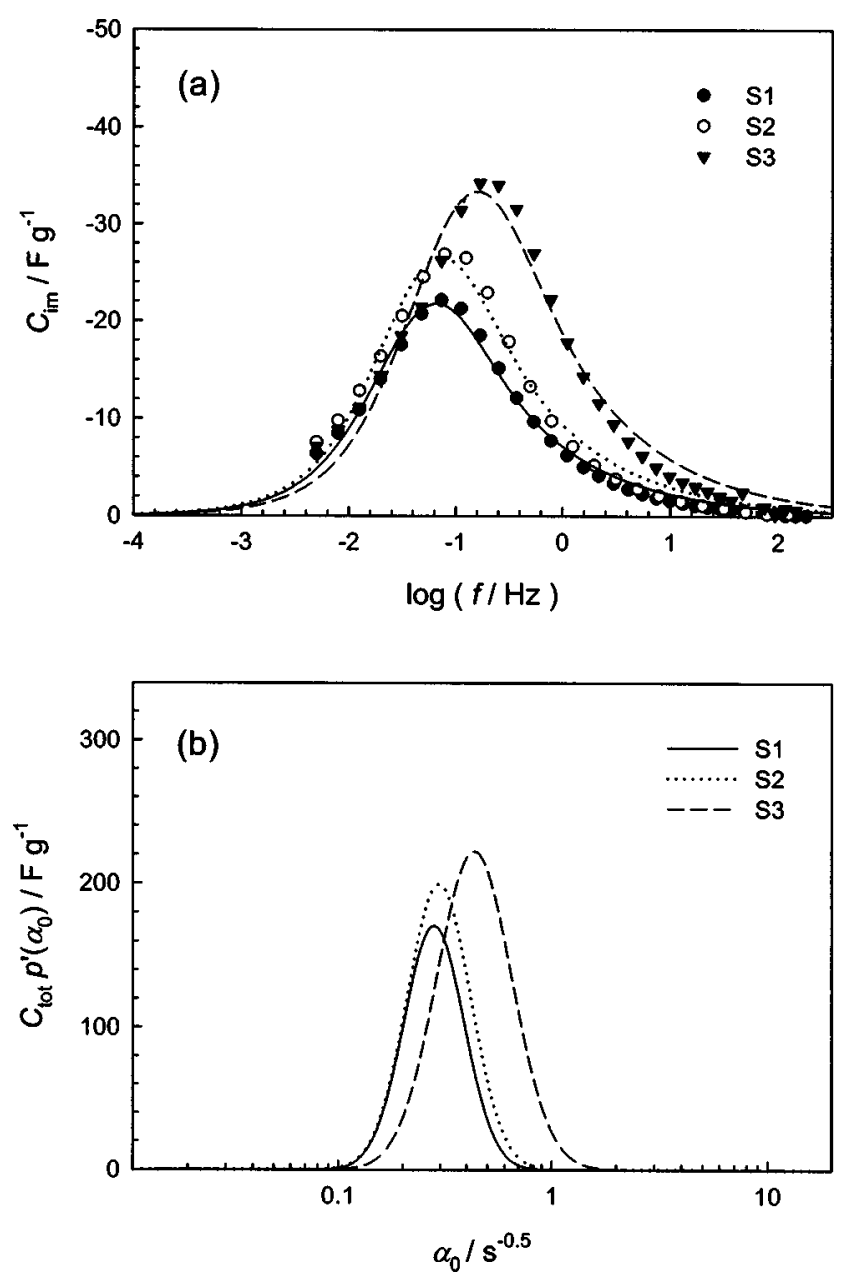

Figure 7. (a) Imaginary capacitance plots converted from the experimental impedance data for silica-sol-templated carbon electrodes. The lines indicate the imaginary capacitance curves that are best-fitted to the experimental data points. (b) $C_{\text {tot }} p^{\prime}\left(\alpha_{0}\right)$ curves derived from the best-fitted imaginary capacitance profiles.

tion data confirm this prediction. Also, the $C_{\text {tot }}$ values are in good accordance with those obtained from the cyclic voltammetry (S1, 59 $\mathrm{F} \mathrm{g}^{-1}$; S2, $70 \mathrm{~F} \mathrm{~g}^{-1} ; \mathrm{S} 3,96 \mathrm{~F} \mathrm{~g}^{-1}$ ). The pore structure of the three carbons can also be compared using the $\alpha_{0}$ distribution curves shown in Fig. 7b, where it is seen that the characteristic penetrability becomes higher with an increase in pore volume. This feature may be explained by the assumption that both the electrolyte conductivity $(\kappa)$ in the pores and double-layer capacitance per unit area $\left(C_{\mathrm{d}}\right)$ are largely the same for the three carbons, as their pore size is comparable to each other $(c a .9 \mathrm{~nm})$. The higher population of larger

Table II. The best-fitted EDLC parameters obtained by complex capacitance analysis and nitrogen gas adsorption data for silicasol-templated mesoporous carbons.

S1

S2

S3

$\begin{array}{lccc}C_{\text {tot }}\left(\mathrm{F} \mathrm{g}^{-1}\right) & 60.4 \pm 0.9 & 74.6 \pm 3.1 & 98.0 \pm 2.3 \\ \sigma & 0.141 \pm 0.014 & 0.149 \pm 0.037 & 0.176 \pm 0.019 \\ \alpha_{0}^{*}\left(\mathrm{~s}^{-0.5}\right) & 0.281 \pm 0.007 & 0.297 \pm 0.019 & 0.434 \pm 0.011 \\ A_{\text {BET }}\left(\mathrm{m}^{2} \mathrm{~g}^{-1}\right) & 457 & 626 & 746 \\ D_{\text {pore,aver. }}(\mathrm{nm}) & c a .9 & c a .9 & c a .9 \\ V_{\text {pore }}\left(\mathrm{cm}^{3} \mathrm{~g}^{-1}\right)^{\mathrm{a}} & 0.68 & 1.08 & 1.50\end{array}$

${ }^{\text {a }}$ Pore volume calculated by BJH method. 
$\alpha_{0}$ but with a comparable pore size manifests itself in that the pore length becomes shorter with an increase in pore volume. It is not difficult to explain this in that the distance between pores is shorter as the pore volume increases.

\section{Conclusion}

The complex capacitance for a cylindrical pore and multiple pores is theoretically derived and the penetrability $\left(\alpha_{0}\right)$ distribution obtained either by log-normal assumption or DFT. The complex capacitance analysis is made on two sets of porous carbon electrodes: one series of carbons differs in the pore size in 1-2 $\mathrm{nm}$ range and the other differs in the pore volume but with a comparable pore size $(c a .9 \mathrm{~nm})$. It is found that the total capacitance and rate capability of the porous carbon electrodes can be easily estimated by displaying the experimental impedance data in the imaginary capacitance plots. This is the strong point of this new methodology over conventional ones where analyses were made employing Nyquist impedance plots $\left(-Z_{\mathrm{im}}\right.$ vs. $\left.Z_{\mathrm{re}}\right)$. The pore structure of carbons can also be predicted from the penetrability distribution curves. When the EDLC performance of the two series of porous carbon electrodes is examined using complex capacitance analysis, the results are reasonably matched with those predicted from the gas adsorption data.

\section{Acknowledgment}

This work was supported by KOSEF through the Research Center for Energy Conversion and Storage.

\section{References}

1. H. Shi, Electrochim. Acta, 41, 1633 (1996).

2. D. Qu and H. Shi, J. Power Sources, 74, 99 (1998).

3. C. Lin, J. A. Ritter, and B. N. Popov, J. Electrochem. Soc., 146, 3639 (1999).

4. S. Yoon, J. Lee, T. Hyeon, and S. M. Oh, J. Electrochem. Soc., 147, 2507 (2000).

5. M. Endo, T. Maeda, T. Takeda, Y. J. Kim, K. Koshiba, H. Hara, and M. S. Dresselhaus, J. Electrochem. Soc., 148, A910 (2001)

6. J. H. Jang, S. Han, T. Hyeon, and S. M. Oh, J. Power Sources, 123, 79 (2003).
7. K. Honda, T. N. Rao, D. A. Tryk, A. Fujishima, M. Watanabe, K. Yasui, and H. Masuda, J. Electrochem. Soc., 148, A668 (2001).

8. H.-K. Song, Y.-H. Jung, K.-H. Lee, and L. H. Dao, Electrochim. Acta, 44, 3513 (1999).

9. H.-K. Song, H.-Y. Hwang, K.-H. Lee, and L. H. Dao, Electrochim. Acta, 45, 2241 (2000).

10. H.-K. Song and K.-H. Lee, Mater. Res. Soc. Symp. Proc., 699, R7.7 (2002).

11. F. Lufrano, P. Staiti, and M. Minutoli, J. Power Sources, 124, 314 (2003).

12. P. L. Taberna, P. Simon, and J. F. Fauvarque, J. Electrochem. Soc., 150, A292 (2003).

13. R. de Levie, in Advances in Electrochemistry and Electrochemical Engineering, Vol. VI, P. Delahay, Editor, p. 329, John Wiley \& Sons, New York (1967).

14. H. Keiser, K. D. Beccu, and M. A. Gutjahr, Electrochim. Acta, 21, 539 (1976)

15. J.-P. Candy, P. Fouilloux, M. Keddam, and H. Takenouti, Electrochim. Acta, 26, 1029 (1981).

16. J.-P. Candy, P. Fouilloux, M. Keddam, and H. Takenouti, Electrochim. Acta, 27, 1585 (1982).

17. K. Eloot, F. Debuyck, M. Moors, and A. P. van Peteghem, J. Appl. Electrochem., 25, 326 (1995)

18. K. Eloot, F. Debuyck, M. Moors, and A. P. van Peteghem, J. Appl. Electrochem., 25, 334 (1995)

19. P. H. Nguyen and G. Paasch, J. Electroanal. Chem., 460, 63 (1999).

20. W. G. Pell, B. E. Conway, W. A. Adams, and J. de Oliveira, J. Power Sources, 80 134 (1999).

21. R. Kotz and M. Carlen, Electrochim. Acta, 45, 2483 (2000).

22. W. G. Pell and B. E. Conway, J. Electroanal. Chem., 500, 121 (2001).

23. B. E. Conway and W. G. Pell, J. Power Sources, 105, 169 (2002).

24. C.-H. Kim, S.-I. Pyun, and H.-C. Shin, J. Electrochem. Soc., 149, A93 (2002).

25. K. Kinoshita, Carbon: Electrochemical and Physicochemical Properties, p. 12 John Wiley \& Sons, New York (1988).

26. H. Bode, Lead-Acid Batteries, p. 144, Wiley-Interscience, New York (1977).

27. D. D. Macdonald and M. Urquidi-Macdonald, J. Electrochem. Soc., 132, 2316 (1985).

28. M. Urquidi-Macdonald, S. Real, and D. D. Macdonald, J. Electrochem. Soc., 133, 2018 (1986)

29. W. L. Briggs and V. E. Henson, The DFT: An Owner's Manual for the Discrete Fourier Transform, The Society for Industrial and Applied Mathematics, Philadelphia, PA (1995)

30. E. O. Brigham, The Fast Fourier Transform and Its Applications, Prentice Hall, Englewood Cliffs, NJ (1988).

31. J. Lee, S. Yoon, T. Hyeon, S. M. Oh, and K. B. Kim, Chem. Commun. (Cambridge), 21, 2177 (1999). 